\title{
Investigation of Battery Charge and Discharge Behavior Based on Measured Field Data
}

\author{
David Theuerkauf $^{1}$, Jordan Torrealba ${ }^{2}$, Andrew Swingler ${ }^{3 *}$ \\ ${ }^{123}$ Faculty of Sustainable Design Engineering, University of Prince Edward Island, Charlottetown, Canada \\ *aswingler@upei.ca
}

\begin{abstract}
Work was completed for Delta-q based off the NSERC Engage grant awarded to Dr. Andrew Swingler. Bespoke battery monitors were installed on $48 \mathrm{~V}$ golf cart battery packs around Prince Edward Island and monitored multiple parameters of performance on a five second interval. These monitors captured complete charge and discharge cycles of multiple charger types and discharge profiles from varied real-world courses. This data was plotted and analyzed for events and trends in the datasets. Common characteristics were found between all chargers, and interesting events were noted. This led to reasoning what the optimal traits of a golf cart charger are for longevity of batteries and end user satisfaction. From observing this data, an opportunity for improvement of battery test bench set ups was found. It was found that the dynamics of lead acid battery performance was not accounted for with existing test bench set ups, and this could substantially impact the batteries performance and longevity in real world applications.
\end{abstract}

Word count: 160 